Pacific Journal of Mathematics

SUPERSIMPLE SETS AND THE PROBLEM OF EXTENDING A 


\section{SUPERSIMPLE SETS AND THE PROBLEM OF EXTENDING A RETRACING FUNCTION}

\section{T. G. McLaughlin}

An infinite set $A$ of natural numbers is called regressive if there is a (non-repeating) sequential ordering $a_{0}, a_{1}, \cdots$ of $A$ and a partial recursive function $f$ such that $A \cong \operatorname{domain}(f)$, $f\left(a_{0}\right)=a_{0}$, and $(\forall n)\left[f\left(a_{n+1}\right)=a_{n}\right]$; in case $a_{0}, a_{1}, \cdots$ is the natural ordering of $A$ by increasing size of elements, $A$ is called retraceable and $f$ is said to retrace $A$. It sometimes happens that a retraceable set does not admit an everywhere-defined retracing function, or that it does not admit a finite-to-one retracing function. Question: does there exist an infinite set $A$ of natural numbers such that $A$ is retraced both by a total recursive function and by a finite-to-one partial recursive function, but not by a function which is both total recursive and finite-toone? Via some theorems relating to D. A. Martin's notions of supersimple and superimmune sets of natural numbers, a strongly affirmative result is obtained.

1. Introduction. Let $N$ denote the set of all natural numbers. In [3], Martin has termed supersimple any co-infinite $\Sigma_{1}^{0}$ subset $S$ of $N$ for which there does not exist a two-place total recursive function $f(x, y)$ with the properties: (i) $f(x, y)$ is characteristic (i.e., it maps $N$ into $\{0,1\})$; (ii) for each pair of distinct natural numbers $x_{1}$ and $x_{2}$, the sets $\left\{y \mid f\left(x_{1}, y\right\}=0\right\}$ and $\left\{y \mid f\left(x_{2}, y\right)=0\right\}$ are finite and disjoint; and (iii) for every $x$, the set $(N-S) \cap\{y \mid f(x, y)=0\}$ is nonempty. Martin further suggests ([3, p. 306, footnote 2]) that a (not necessarily co-r.e.) set $I \subset N$ be called superimmune provided that $I$ is infinite and that there is no two-place total recursive characteristic function $f(x, y)$ such that the sets $\{y \mid f(x, y)=0\}, x=0,1,2$, $\cdots$, are mutually disjoint (but not necessarily finite) and satisfy $\{y \mid f(x, y)=0\} \cap I \neq \varnothing$ for all $x$. However, as we shall see, the adoption of that terminology would require the admission of supersimple sets having non-superimmune complements, which would not be in keeping with traditional recursion-theoretic nomenclature. Therefore we shall designate as strongly superimmune those sets which Martin recommended calling superimmune; and we shall say that an infinite set $I$ is superimmune if its complement $S$ (not necessarily a $\Sigma_{1}^{0}$ set) admits no total recursive function $f(x, y)$ satisfying (i), (ii) and (iii) above. If $S$ is $\Sigma_{0}^{1}$ and has a strongly superimmune complement, we shall say that $S$ is strongly supersimple.

The following theorem, due originally to Martin, is proved in [4]: 
THEOREM 1. An infinite set $I \subset N$ is strongly superimmune $\Leftrightarrow$ no infinite subset of $I$ can be retraced by a total recursive function.

A partial function $f: K \rightarrow N, K \subseteq N$, is downward $\Leftrightarrow_{d f}$ range $(f) \subseteq$ domain $(f)$ and $(\forall x)[x \in$ domain $(f) \Rightarrow f(x) \leqq x]$. If $f$ is a downward partial function, then for each $x \in \operatorname{domain}(f)$ we denote by $f^{*}(x)$ the least number $n$ such that $f^{n+1}(x)=f^{n}(x)$. (Here $f^{0}(x)={ }_{d f} x, f^{n+1}(x)=$ ${ }_{d f} f\left(f^{n}(x)\right)$.) More generally, if $f$ has the property that range $(f) \subseteq$ domain $(f)$ and $(\forall x)\left[x \in\right.$ domain $\left.(f) \Rightarrow(\exists n)\left(f^{n+1}(x)=f^{n}(x)\right)\right]$, then we say that $f$ is grounded and we denote by $f^{*}(x)$ the least $n$ such that $f^{n+1}(x)=f^{n}(x)$.

In case $I$ is $\Sigma_{2}^{0}$, Theorem 1 can be strengthened as follows:

THEOREM 1*. Suppose that $I \subseteq N$ is infinite, is $\Sigma_{2}^{0}$, and is not strongly superimmune. Then there exist an infinite set $J \subseteq I$ and a pair of functions $g^{\prime}$ and $g$ such that $g^{\prime}$ is partial recursive and finiteto-one, $g$ is total recursive and downward, $g$ is an extension of $g^{\prime}, g^{\prime}$ retraces $J$, and $J$ is the only infinite set retraced by $g$.

A proof of Theorem $1^{*}$ will be given in $\S 2$. In $\S 3$ we shall prove that a form of Theorem $1^{*}$ having a weaker conclusion is optimal with respect to the arithmetical hierarchy, and indicate how to prove the following natural analogue of Theorem 1:

THEOREM 3. An infinite set $I \subseteq N$ is superimmune $\Leftrightarrow$ there is no infinite subset $J$ of $I$ such that $J$ can be retraced by a finite-to-one total recursive function.

In $\S 4$, we apply material from [6] to establish the distinction between superimmunity and strong superimmunity for co-r.e. sets:

THeOREM 4. There exists a supersimple set $S$ such that $S$ is not strongly supersimple.

Our interest in Theorem 4 stems not so much from its character as a structural classification theorem as from the fact that when combined with Theorem $1^{*}$ it provides an easy proof of the following new result on extensibility of retracing functions:

THEOREM 5. There exist an infinite set $R \subset N$ and a pair of functions $f$ and $g$ such that

(5 i) $g$ is a downward, total recursive function which retraces $R$ and no other infinite set,

(5 ii) $f$ is a finite-to-one, partial recursive restriction of $g$ which retraces $R$, and

(5 iii) no infinite subset $T$ of $R$ can be arranged in a sequence 
$\left\{t_{i}\right\}_{i=0}^{\infty}$ in such a way that $\left\{t_{i}\right\}_{1=0}^{\infty}$ admits a finite-to-one, total recursive regressing function.

Proof. Applying Theorem 4, let $S$ be a supersimple set whose complement is not strongly superimmune. By Theorem $1^{*}$, there exist an infinite set $I \cong N-S$ and a total recursive, downward function $g$ such that $(\alpha) I$ is the unique infinite set retraced by $g$ and $(\beta) I$ is retraced by some finite to one, partial recursive restriction $f$ of $g$. Choosing such an $I$, such a $g$, and such a restriction $f$ of $g$ gives us (5 i) and (5 ii) with $R=I$. Keeping $R=I, I$ as just chosen above, we shall establish (5 iii) by contradiction. Suppose that $T \cong R, T$ is infinite, and $T$ is regressive via some finite-to-one, total recursive function. Let $h$ be a particular finite-to-one, total recursive function and $\left\{t_{i}\right\}_{i=0}^{\infty}$ a particular ordering of $T$ into a (non-repeating) sequence such that $h\left(t_{0}\right)=t_{0}$ and $(\forall n)\left[h\left(t_{n+1}\right)=t_{n}\right]$. We shall replace $h$ by a finite-to-one, total recursive function $k$ such that $k\left(t_{0}\right)=t_{0},(\forall n)\left[k\left(t_{n+1}\right)=\right.$ $\left.t_{n}\right]$, and $(\forall x)(\exists n)\left[k^{n+1}(x)=k^{n}(x)\right]$. To do this, we first note that for any given $x$ there are just two ways in which it can fail to be the case that $(\exists n)\left[h^{n+1}(x)=h^{n}(x)\right]$ : either (a) $(\exists n)(\exists m)\left[m \geqq n+2\right.$ and $h^{m}(x)=$ $h^{n}(x)$ and $h^{n+1}(x) \neq h^{n}(x)$ ] (i.e., the $h$-orbit of $x$ contains a proper loop), or (b) $(\forall n)(\forall m)\left[n \neq m \Rightarrow h^{n}(x) \neq h^{m}(x)\right]$ (i.e., the $h$-orbit of $x$ is an infinite "splinter" in the sense of [6]). The elimination of failures of type (a) presents no problem. To eliminate failures of type (b), we take advantage of the fact that the complement of the supersimple set $S$ does not include any infinite $\sum_{1}^{0}$ set and hence, in particular, does not include any infinite set of the form $\left\{y \mid(\exists n)\left[y=h^{n}\left(x_{0}\right)\right]\right\}$. It follows that for each $x \in N$, exactly one of three conditions holds: (a') same as condition (a) above, $\left(\mathrm{b}^{\prime}\right)\left\{h^{n}(x) \mid n \in N\right\} \cap S \neq \varnothing$, or $\left(\mathrm{c}^{\prime}\right)(\exists n)\left[h^{n+1}(x)=\right.$ $\left.h^{n}(x)\right]$. Moreover, we can effectively determine (uniformly in $x$ ) which one of $\left(\mathrm{a}^{\prime}\right),\left(\mathrm{b}^{\prime}\right),\left(\mathrm{c}^{\prime}\right)$ holds, by simultaneously enumerating $S$ and $\left\{h^{n}(x) \mid n \epsilon\right.$ $N\}$. Thus we are able to define $k$ as follows:

$$
k(x)=\left\{\begin{array}{l}
h(x), \text { if }\left(\mathrm{c}^{\prime}\right) \text { holds for } x ; \\
0, \quad \text { if }\left(\mathrm{c}^{\prime}\right) \text { does not hold for } x \text { and } \neg(\exists z)[z<x \text { and } \\
\left.\quad\left(\mathrm{c}^{\prime}\right) \text { holds for } z\right] ; \\
\text { the largest } z \text { such that } z<x \text { and }\left(\mathrm{c}^{\prime}\right) \text { holds for } z, \\
\text { if }\left(\mathrm{c}^{\prime}\right) \text { does not hold for } x \text { and }(\exists z)\left[z<x \text { and }\left(\mathrm{c}^{\prime}\right)\right. \\
\text { holds for } z] .
\end{array}\right.
$$

It is easily verified that $k$, so defined, is a finite-to-one, total recursive, grounded function such that $k\left(t_{0}\right)=t_{0}$ and $(\forall n)\left[k\left(t_{n+1}\right)=t_{n}\right]$. (In particular, as regards finite-to-one, note that for any $y$ there can be only finitely many $x$ such that $\neg(\exists z)\left[y<z<x\right.$ and $\left(\mathrm{c}^{\prime}\right)$ holds for $\left.z\right]$.) We 
next replace $k$ by a total recursive, grounded, finite-to-one function $l$ such that $l\left(t_{0}\right)=t_{0}$ and $(\forall n)\left[l\left(t_{n+1}\right)=t_{n}\right]$ and $(\forall x)\left[l(x)=x \Rightarrow x=t_{0}\right]$. The construction of $l$ from $k$ is very easy:

$$
l(x)=\left\{\begin{array}{l}
k(x), \text { if } k k^{*}(x)(x)=t_{0} ; \\
\text { the least } z \\
\text { such that } k^{*}(z)=x \text { and } k^{x}(z)=t_{0}, \\
\text { if } k k^{*}(x)(x) \neq t_{0} .
\end{array}\right.
$$

Now define $j(x, y)=0$ or 1 according as $l^{*}(y)=x$ or $l^{*}(y) \neq x$; then $j$ is a total recursive characteristic function such that the sets $\{y \mid j(x$, $y)=0\}$ are finite and mutually disjoint and, for every $x,\{y \mid j(x, y)=$ $0\} \cap T \neq \varnothing$. But $T \subseteq R \subseteq N-S$, from which it follows that $S$ is not supersimple: contradiction. (5 iii) follows for $R$, and the proof of Theorem 5 is complete.

Comment. It is known ([5, Theorem 5.2]; [4, Lemma 3]) that if $J$ is the unique infinite set retraced by some partial recursive, finiteto-one function, then $J$ has degree of unsolvability $\leqq 0^{\prime}$. So, in particular, the set $R$ of Theorem 5 has degree $\leqq 0^{\prime}$.

2. Proof of Theorem $1^{*}$. To begin with, we note that it essentially suffices to prove Theorem $1^{*}$ under the assumption that $I$ is infinite, not strongly superimmune, and of degree $\leqq 0^{\circ}$. For suppose the theorem has been established subject to that stronger assumption. Let $I_{0}$ be a $\Sigma_{2}^{0}$ subset of $N$ satisfying the hypotheses of Theorem $1^{*}$. Then $I_{0}$, being $\Sigma_{2}^{0}$, is the range of a function $\nu: N \rightarrow N$ such that $\nu$ is of degree $\leqq$ $0^{\prime}$. Let $f(x, y)$ be a total recursive characteristic function witnessing the failure of $I_{0}$ to be strongly superimmune, and such that $(\forall x)\left[I_{0} \cap\right.$ $\{y \mid f(x, y)=0\}$ is infinite]. (Clearly, such an $f$ must exist, if $I_{0}$ is not strongly superimmune.) It is clear that using $\nu$ and $f$ we can define a function $\xi: N \rightarrow N$ such that (i) $\xi$ is strictly increasing, (ii) $\xi$ is of degree $\leqq 0^{\prime}$, and (iii) $(\forall n)\left[\xi(n) \in I_{0} \cap\{y \mid f(n, y)=0\}\right]$. Hence, if $E=$ range $(\xi)$ then $E$ is an infinite subset of $I_{0}$ such that (iv) $E$ is of degree $\leqq 0^{\prime}$ and (v) $E$ satisfies the hypotheses of Theorem $1^{*}$. It follows that $E$, and therefore also $I_{0}$, satisfies the conclusion of Theorem $1^{*}$. We now proceed to show that if $I$ is an infinite set of degree $\leqq 0^{\prime}$ which is not strongly superimmune, then $I$ satisfies the conclusion of Theorem $1^{*}$. Let $f(x, y)$ witness the failure of $I$ to be strongly superimmune. As is well-known, since $I$ is of degree $\leqq 0^{\prime}$ there exists a recursive characteristic function $h(s, x)$ such that $(\forall x)\left[\lim _{s \rightarrow \infty} h(s\right.$, $x$ ) exists and $=c_{I}(x)$ ], where $c_{I}$ is the characteristic function of $I$ (i.e., $c_{I}(x)=0$ if $x \in I$ and $c_{I}(x)=1$ if $\left.x \notin I\right)$. We shall construct the required function $g$ by stages, using a sequence $\left\{\Lambda_{i}\right\}_{i=0}^{\infty}$ of "markers"; the con- 
struction will be such that at the end of stage $s$ there is a number $t(s) \leqq s$ such that exactly the first $t(s)+1$ markers, $\Lambda_{0}, \Lambda_{1}, \cdots, \Lambda_{t(s)}$, are associated with numbers. (When the construction has been completely described, it will be clear that $t(s)$ is a recursive function.) No marker will ever be associated with more than one number at a time; nor will any number ever have more than one marker-associate at a time. We shall denote by $\lambda_{u}^{s}$ the number with which $\Lambda_{u}$ is associated at the beginning of Stage $s+1$, for each $u \leqq t(s)$. The construction proceeds thus:

Stage 0. Associate $\Lambda_{0}$ with $a_{0}$, where $a_{0}$ is the least $a \in I$ such that $f(0, a)=0$. Let $v_{0}$ be the least number $v$ such that $v>0$ and $(\forall y)\left[f(v, y)=0 \Rightarrow y>a_{0}\right]$. Associate $\Lambda_{1}$ with $(\mu n)\left[f\left(v_{0}, n\right)=0\right]$. Set $g_{0}=\left\{(x, x) \mid x<a_{0}\right.$ or $\left.f(0, x)=0\right\} \cup\left\{\left(x, a_{0}\right) \mid f\left(v_{0}, x\right)=0\right\}$; then pass to Stage 1.

Stage $s+1$. Suppose that precisely the markers $\Lambda_{0}, \Lambda_{1}, \cdots, \Lambda_{t(s)}$ are associated with numbers at the end of Stage $s$, where $t(s)$ is a number $\leqq s$ whose exact value is known at the end of Stage $s$. (Note, in particular, that $t(0)=1$.) There are three cases.

Case I. $(\forall z)\left[0<z \leqq t(s) \Rightarrow h\left(s, \lambda_{z}^{s}\right)=0\right]$. In this case, let $r_{0}$ be the least $r$ such that (i') no member of $\{y \mid f(r, y)=0\}$ belongs to the domain of $g_{s}$, and (ii') $(\forall y)\left[f(r, y)=0 \Rightarrow y>\lambda_{t(s)}^{s}\right]$. Let $\widetilde{g}_{s+1}=g_{s} \cup$ $\left\{\left(x, \lambda_{t(s)}^{s}\right) \mid f\left(r_{0}, x\right)=0\right\}$; and define $g_{s+1}=\widetilde{g}_{s+1} \cup\left\{\left(t, a_{0}\right) \mid t \leqq s\right.$ and $t \notin$ domain $\left.\left(\widetilde{g}_{s+1}\right)\right\}$. Associate $\Lambda_{t(s)+1}$ with $u_{0}$, where $u_{0}$ is the least number $u$ such that $f\left(r_{0}, u\right)=0$. Letting $t(s+1)=t(s)+1$, proceed to Stage $s+2$.

Case II. Case I does not obtain, and if $z_{0}=$ the least $z$ such that $0<z \leqq t(s)$ and $h\left(s, \lambda_{z}^{s}\right) \neq 0$ then $(\exists w)[w \leqq s$ and $h(s, w)=0$ and $\left.(\forall k)\left[f\left(k, \lambda_{z}^{s}\right)=0 \Rightarrow f(k, w)=0\right]\right]$. Let $z_{0}$ be the least $z, 0<z \leqq t(s)$, such that $h\left(s, \lambda_{z}^{s}\right)=1$. Disassociate the markers $\Lambda_{z_{0}}, \Lambda_{z_{0}+1}, \cdots, \Lambda_{t(s)}$ from their current associates $\lambda_{z_{0}}^{s}, \lambda_{z_{0}+1}^{s}, \cdots, \lambda_{(t s)}^{s}$. Let $w_{0}$ be the uniquely determined number $w$ such that $f\left(w, \lambda_{z_{0}}^{s}\right)=0$. (The existence of $w_{0}$ will be clear once our description of the construction is complete.) Let $u_{0}$ be the least number $u$ such that $f\left(w_{0}, u\right)=0$ and $h(s, u)=0$. Re-associate $\Lambda_{z_{0}}$ by associating it with $u_{0}$ (i.e., set $\lambda_{z_{0}}^{s+1}=u_{0}$ ). Set $g_{s+1}=g_{s} \cup\left\{\left(t, a_{0}\right) \mid t \leqq s\right.$ and $t \notin$ domain $\left.\left(g_{s}\right)\right\}$. Letting $t(s+1)=z_{0}$, proceed to Stage $s+2$.

Case III. Neither of Cases I, II obtain. Make no marker changes. Set $g_{s+1}=g_{s}$, and go to Stage $s+2$.

To complete the construction of $g$, we define $g=\bigcup_{s \in N} g_{s}$. To see that $g$, as so defined, is a partial recursive function, we merely note 
that at every point in the construction where a specific number needs to be computed or a specific alternative needs to be decided, the computation or decision in question can be made effectively, uniformly in all parameters involved. For example, if at Stage $s+1$ we assume that we know a computation procedure $\mathscr{P}(s+1)$ for determining the least number $q$ such that $\{y \mid f(q, y)=0\} \cap$ domain $\left(g_{s}\right)=\varnothing$ (the existence of such a $q$ being obvious from the description of Stages 0 and $s+1)$, and if we also assume that the value of $\lambda_{t(s)}^{s}$ is known, then in Case I we can uniformly effectively determine $r_{0}$ from $\mathscr{P}(s+1)$ and $\lambda_{t(s)}^{s}$. But, in view of our description of Stage $s+1$ in its totality, it is clear that we can assume inductively that we have such knowledge of domain $\left(g_{s}\right)$ and of $\lambda_{t(s)}^{s}$; in particular, we obviously have it for domain $\left(g_{0}\right)$ and $\lambda_{0}^{0}$. Again, it is clear from the statement of the construction for the general stage $s+1$ that $(\forall x)\left[x<s \Rightarrow\left(g_{s}(x)\right.\right.$ is defined and $\left.\left.g_{t}(x) \leqq x\right)\right]$; thus $g=\bigcup_{s \in N} g_{s}$ is total recursive and downward. We next verify, by mathematical indution on $s$, that $\lim _{s \rightarrow \infty} \lambda_{u}^{s}$ exists for all $u$ (i.e., that each marker $\Lambda_{u}$ eventually attains permanent association with some number) and that $\lim _{s \rightarrow \infty} \lambda_{u}^{s} \in I$. To begin with, since Cases I and II of Stage $s+1$ concern only $z$ which are greater than zero, we see at once that $\lambda_{0}^{s}=a_{0}$ for all values of $s$. Suppose $\lim _{s \rightarrow \infty} \lambda_{u}^{s}$ exists and belongs to $I$ for all $u<v$; and let $s_{0}$ be the smallest number such that $(\forall u<v)\left(\forall s \geqq s_{0}\right)\left[\lambda_{u}^{s}\right.$ is defined and $\left.=\lim _{s \rightarrow>\infty} \lambda_{u}^{s}\right]$. Let $s_{1} \geqq s_{0}$ be chosen so that $h\left(s, \lambda_{u}^{s}\right)=0$ for all $s \geqq s_{1}$ and all $u<v$. Then $\Lambda_{v}$ becomes associated with some number $m_{0}$ via Case I at Stage $s_{1}+1$, if it is not already attached by the end of Stage $s_{1}$; here $f\left(p_{0}, m_{0}\right)=0$ holds for a certain (uniquely determined) number $p_{0}$. If, subsequent to Stage $s_{1}$, say at Stage $s_{1}+w+1, \Lambda_{v}$ loses its association with $m_{0}$, it must be because Case II applies with $h\left(s_{1}+w, m_{0}\right)=1$. But in that event, $\Lambda_{v}$ is immediately re-associated with a new number $m_{1}$ satisfying $f\left(p_{0}\right.$, $\left.m_{1}\right)=0$ and $h\left(s_{1}+w, m_{1}\right)=0$. If $\Lambda_{v}$ later loses its association with $m_{1}$, it is due to another application of Case II, and again $\Lambda_{v}$ is at once re-associated, this time with some number $m_{2}$ satisfying $f\left(p_{0}, m_{2}\right)=0$ and $h\left(s_{2}, m_{2}\right)=0$ where $s_{2}$ is the stage in question. And so on. Since $\left\{y \mid f\left(p_{0}, y\right)=0\right\} \cap I \neq \varnothing$ and $(\forall y)\left[\lim _{s \rightarrow \infty} h(s, y)=c_{I}(y)\right]$, we see from the description of Case II that this process can lead to only finitely many changes in the value of $\lambda_{v}^{s}$; whence $\lim _{s \rightarrow \infty} \lambda_{v}^{s}$ exists and lies in I. So, by induction, $\lim _{s \rightarrow \infty} \lambda_{u}^{s}$ exists and lies in $I$ for all $u$. We denote $\lim _{s \rightarrow \infty} \lambda_{u}^{s}$ by $\lambda_{u}$. It is evident from the construction that $(\forall u)\left[\lambda_{u}<\lambda_{u+1}\right.$ and $g\left(\lambda_{u+1}\right)=\lambda_{u}$; also, we clearly have $g\left(\lambda_{0}\right)=g\left(a_{0}\right)=a_{0}=$ $\lambda_{0}$. Thus, setting $J=\left\{\lambda_{n} \mid n \in N\right\}$, we have that $J$ is an infinite subset of $I$ retraced by $g$. We claim that $g$ can retrace no other infinite set. For suppose $\neg(\exists n)\left(x=\lambda_{n}\right]$. Then there is a stage $s$ such that $s^{\prime} \geqq$ $s \Rightarrow(\forall n)\left[x \neq \lambda_{n}^{s^{\prime}}\right]$. (For, our description of the construction makes it clear that no number $x$ can satisfy both $(\exists u)\left[x=\lambda_{e_{1}}^{u}\right]$ and $(\exists v)\left[x=\lambda_{e_{2}}^{v}\right]$ 
with $e_{1} \neq e_{2}$.) But it is trivial to show by induction on $s$ that if $(\forall n)\left[x \neq \lambda_{n}^{s}\right]$, then $y \in$ domain $\left(g_{s+1}\right)$-domain $\left(g_{s}\right) \Rightarrow \neg(\exists w)\left[g_{s+1}^{w}(y)=x\right]$. Thus if $x$ belongs to an infinite set retraced by $g$ while yet $x \notin\left\{\lambda_{n} \mid n \in\right.$ $N\}$, then there is a stage $s$ of the construction such that $(\forall n>$ $0)(\exists y)\left[g_{s+1}^{n}(y)=x<g_{s+1}^{n-1}(y)<\cdots<g_{s+1}(y)<y\right]$. From the definition of $g_{s}$, however, using induction on $s$, we see that this cannot be the case. Hence $J=\left\{\lambda_{n} \mid n \in N\right\}$ is the only infinite set retraced by $g$. It remains only to prove that there exists a finite-to-one partial recursive restriction $g^{\prime}$ of $g$ which retains the property of retracing $J$. The existence of such a function $g^{\prime}$, however, follows from [5, Theorem 8], since it is easily verified that $\left\{\lambda_{n} \mid n \in N\right\}$ has degree of unsolvability $\leqq 0^{\prime}$ (Simply note that $\lambda_{n}^{s}$ is a recursive function of $n$ and $s$ if we set $\lambda_{n}^{s}=0$ whenever $\Lambda_{n}$ is unattached at the end of Stage s.) That completes the proof of Theorem $1^{*}$.

3. Optimality of Theorem $1^{*}$ and Proof of Theorem 3. The following result shows that the $\Sigma_{2}^{0}$ classification of $I$ in Theorem $1^{*}$ cannot be carried any further within the standard arithmetical hierarchy, even if we drop the requirement that $J$ be the only infinite set retraced by $g^{\prime}$.

THEOREM 2. There exists an infinite $I_{2}^{0}$ subset $I$ of $N$ such that

(2i) I is the unique infinite set retraced by a total recursive, downward function $g$;

(2 ii) $I$ is not strongly superimmune; and

(2 iii) no infinite set $J \subseteq I$ can be regressed by a finite-to-one partial recursive function.

Proof. By [1, Theorem 4.14(2)], there exists a total recursive function $g$ and an infinite $\Pi_{2}^{0}$ set $I \leqq N$ such that $I$ has degree $0^{\prime \prime}$ and $I$ is the unique infinite set retraced by $g$; clearly we may suppose also that $g$ is downward. Next, if we define $f(x, y)=0 \Leftrightarrow g^{*}(y)=x$ and $f(x, y)=1$ otherwise, then plainly $f$ witnesses the failure of $I$ to be strongly superimmune. (This is just the proof of the easier half of Theorem 1.) Finally, suppose $J \subseteq I, J$ infinite, and $\widetilde{g}$ regresses $J$ where $\widetilde{g}$ is a finite-to-one partial recursive function. Then, by a standard argument, we see that there exists an infinite set $H \subseteq J$ such that $H$ is retraced by a finite-to-one partial recursive function $\zeta$. Let $\widetilde{\zeta}$ be defined as follows:

$$
(x, y) \in \widetilde{\zeta} \Leftrightarrow_{d f}(x, y) \in \zeta \text { and }(\exists z)\left[g^{z}(x)=y\right] .
$$

Then $\tilde{\zeta}$ is a finite-to-one partial recursive function such that every infinite set which it retraces is a subset of $I$. Certainly, $\tilde{\zeta}$ retraces $H$ among (possibly) others. Hence by [5, Theorem 2], $I$ has 
an infinite (retraceable) subset of degree strictly less than $0^{\prime \prime}$. But therefore also $I$ has degree $ફ 0^{\prime \prime}$, since $I$ is retraceable and so is reducible to all of its infinite subsets. Since this contradicts our choice of $I$, Theorem 2 is proven.

We now sketch an argument for Theorem 3. Let $f$ be a finiteto-one, total recursive function which retraces an infinite subset $R$ of $I$. We first replace $f$ by a finite-to-one, total recursive, downward function $g$ which likewise retraces $R$; the procedure for defining $g$ from $f$ is similar to, but less involved than, the procedure used to obtain $k$ from $h$ in our proof of Theorem 5. Setting $r(x, y)=0$ or 1 according as $g^{*}(y)=x$ or $g^{*}(y) \neq x$, we then have $r$ a witness to nonsuperimmunity of $I$. Conversely suppose $g(x, y)$ is a total recursive characteristic function witnessing non-superimmunity of $I$. We modify the construction on p. 258 of [4] as follows, all notation being exactly as in [4]:

\section{Stage 0. Same as Stage 0 on p. 258 of [4].}

Stage $s+1$. Same as Stage $s+1$ on p. 258 of [4], except that at the last step of the stage, instead of placing $(t, 0)$ in $f$ provided $t<s$ and $t$ is not yet in domain $(f)$, we place $(t, k)$ in $f$ just in case $t<s$ and $k<t$ and $t$ is not yet in domain $(f)$ and $f^{*}(k)$ is defined by the end of stage $s$ and $k$ is the largest number $l<t$ for which $f^{*}(l)$ is defined by the end of stage $s$.

As thus defined by stages, $f$ is obviously a partial recursive function. Exactly as in [4], we check that $f$ retraces an infinite subset of $I$. It then readily follows that $f$ is defined for all but finitely many $x \in N$, and that $f$ is finite-to-one. An at-most-finite adjustment of $f$ (to insure totality) then completes the verification of Theorem 3.

Comment. If we asume $I$ to be infinite, non-superimmune, and of degree $\leqq 0^{\prime}$ then, proceeding very much as in our proof of Theorem $1^{*}$, we can find an infinite set $R \subset I$ such that $R$ is the unique infinite set retraced by some finite-to-one total recursive function. We do not know whether this can be improved to the extent of assuming merely that $I$ is infinite, non-superimmune, and $\sum_{2}^{0}$.

4. Proof of Theorem 4. In [6], Young has studied in some detail two special classes of $\sum_{1}^{0}$ sets: the class $\boldsymbol{S H S}$ of "strongly hypersimple" sets, i.e., r.e. sets $S$ such that if $\left\{W_{r(i)}\right\}_{i=0}^{\infty}$ is any recursive sequence of disjoint r.e. sets whose union $=N$ then $(\exists i)\left[W_{r(i)} \cap(N-\right.$ $S)=\varnothing$ ], and the class FSHS of "finitely strongly hypersimple" sets, i.e., r.e. sets $S$ such that if $\left\{W_{r(i)}\right\}_{i=0}^{\infty}$ is any recursive sequence of 
disjoint finite r.e. sets whose union $=N$ then $(\exists i)\left[W_{r(i)} \cap(N-S)=\varnothing\right]$. (Here $\left\{W_{i}\right\}_{i=0}^{\infty}$ is some standard-type recursive enumeration of the class of all r.e. sets.) The following lemma, brought to our attention by Carl Jockusch, establishes the equivalence of Young's definitions to those used in the present paper:

Lemma. $\boldsymbol{S} \in \boldsymbol{F} \boldsymbol{S H S} \Leftrightarrow \boldsymbol{S}$ is supersimple; and $\boldsymbol{S} \in \boldsymbol{S H S} \Leftrightarrow S$ is strongly supersimple.

Proof of Lemma. Suppose $S$ is not supersimple. By Theorem 3, there is a total recursive, finite-to-one function $f$ such that $f$ retraces an infinite subset of $N-S$. There is no loss of generality in assuming $f$ to be downward. But then if $r(x)$ is a recursive function such that $W_{r(x)}=\left\{y \mid f^{*}(y)=x\right\}$, the sequence $\left\{W_{r(i)}\right\}_{i=0}^{\infty}$ witnesses $\boldsymbol{S} \notin \boldsymbol{F S H S}$. By a similar argument, using Theorem 1 instead of Theorem 3, we have $S \in \boldsymbol{S H S} \Rightarrow S$ is strongly supersimple. For the converse implications, suppose, for example, that $\boldsymbol{S} \notin \boldsymbol{F S H S}$; and let $\left\{W_{r(i)}\right\}_{i=0}^{\infty}$ witness that fact. Define $g(x, y)=0$ or 1 according as $y \in W_{r(x)}$ or not (since $N=\bigcup_{n} W_{r(n)}$, this is an effective disjunction); then $g$ witnesses $S$ non-supersimple. Similarly for $\boldsymbol{S H S}$. (This same procedure could be used, instead of Theorems 1 and 3, in the other half of the proof.)

Returning to the proof of Theorem 4, we observe that Young has shown, in Corollary 2.9 of [6], that the difference class $\boldsymbol{F S H S}-\boldsymbol{S H S}$ is nonempty. Hence, by the above Lemma, we have Theorem 4 .

Comments. (1) Martin conjectured in [3] that there exist supersimple sets which are not dense simple, i.e., whose complements (enumerated in order of magnitude) do not eventually dominate any given total recursive function. In a footnote added in press to [3], he remarked that A. H. Lachlan verified this conjecture by demonstrating the existence of $r$-maximal $\Sigma_{1}^{0}$ sets with no dense simple supersets. (A $\Sigma_{1}^{0}$ set is $r$-maximal if its complement is infinite and cannot be nontrivially split by a recursive set.) Lachlan's result appears as Theorem 8 in [2]. Young's method of producing elements of FSHS$\boldsymbol{S H S}$ is to apply the operator $\Phi_{f}$ to a member of $\boldsymbol{F S H S}$, where $f$ is a finite-to-one total recursive function and $\Phi_{f}(A)={ }_{d f}(A \otimes N) \cup \mathbf{U}_{k \in N}(\{k\} \otimes$ $\{x \mid x>f(k)\})$. (Here $\otimes$ denotes Cartesian product.) But it is easily demonstrated that if $f$ is a strictly increasing total recursive function then, for any co-infinite $\Sigma_{1}^{0}$ set $A, \Phi(A)$ is not dense simple. Thus the sets to which we have had recourse in proving Theorem 4 also furnish (when $f$ is strictly increasing) examples of non-dense supersimple sets far removed from the class of $r$-maximal sets. 
(2) It is natural to inquire whether Theorem 4 can be raised to the level of non-embedding: is there a supersimple set with no strongly supersimple extension? R. W. Robinson has constructed a class of nonrecursive r.e. sets none of which has a strongly supersimple extension; however, each member of Robinson's class in non-supersimple.

\section{REFERENCES}

1. C. G. Jockusch, Jr., and T. G. McLaughlin, Countable retracing functions and $\Pi_{2}^{0}$ predicates, Pacific J. Math., 30 (1969), 67-93.

2. A. H. Lachlan, On the lattice of recursively enumerable sets, Trans. Amer. Math. Soc., 130 (1968), 1-37.

3. Donald A. Martin, Classes of recursively enumerable sets and degrees of unsolvability, Z. Math. Logik Grundlagen Math., 12 (1966), 295-310.

4. T. G. McLaughlin, Some remarks on extensibility, confluence of paths, branching properties, and index sets, for certain recursively enumerable graphs, Illinois J. Math., 11 (1967), 257-279.

5. C. E. M. Yates, Arithmetical sets and retracing functions, Z. Math. Logik Grundlagen Math., 13 (1967), 193-204.

6. Paul R. Young, Linear orderings under one-one reducibility, J. Symbolic Logic, 31 (1966), 70-85.

Received November 30, 1970 and in revised form September 1, 1971. Preparation of this paper for publication was partially supported by funds from NSF grant GP 29223. The author wishes to thank the referee for pointing out an overzealous claim in the original proof of Theorem 2 .

The University of Illinois at Urbana 


\section{PACIFIC JOURNAL OF MATHEMATICS}

\section{EDITORS}

\author{
H. SAMELSON \\ Stanford University \\ Stanford, California 94305

\section{R. HoвBY} \\ University of Washington \\ Seattle, Washington 98105
}

\section{J. DugundJI}

Department of Mathematics University of Southern California Los Angeles, California 90007

\author{
RichaRd ARENS \\ University of California \\ Los Angeles, California 90024
}

\section{ASSOCIATE EDITORS}
E. F. BECKENBACH
B. H. NEUMANN
F. WOLF
K. YoshidA

\section{SUPPORTING INSTITUTIONS}

\author{
UNIVERSITY OF BRITISH COLUMBIA \\ CALIFORNIA INSTITUTE OF TECHNOLOGY \\ UNIVERSITY OF CALIFORNIA \\ MONTANA STATE UNIVERSITY \\ UNIVERSITY OF NEVADA \\ NEW MEXICO STATE UNIVERSITY \\ OREGON STATE UNIVERSITY \\ UNIVERSITY OF OREGON \\ OSAKA UNIVERSITY
}

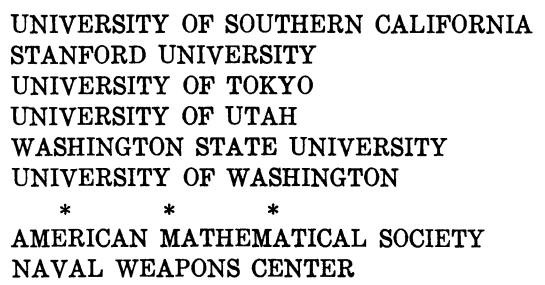

The Supporting Institutions listed above contribute to the cost of publication of this Journal, but they are not owners or publishers and have no responsibility for its content or policies.

Mathematical papers intended for publication in the Pacific Journal of Mathematics should be in typed form or offset-reproduced, (not dittoed), double spaced with large margins. Underline Greek letters in red, German in green, and script in blue. The first paragraph or two must be capable of being used separately as a synopsis of the entire paper. The editorial "we" must not be used in the synopsis, and items of the bibliography should not be cited there unless absolutely necessary, in which case they must be identified by author and Journal, rather than by item number. Manuscripts, in dup icate if possible, may be sent to any one of the four editors. Please classify according to the scheme of Math. Rev. Index to Vol. 39. All other communications to the editors should be addressed to the managing editor, Richard Arens, University of California, Los Angeles, California, 90024.

50 reprints are provided free for each article; additional copies may be obtained at cost in multiples of 50 .

The Pacific Journal of Mathematics is published monthly. Effective with Volume 16 the price per volume (3 numbers) is $\$ 8.00$; single issues, $\$ 3.00$. Special price for current issues to individual faculty members of supporting institutions and to individual members of the American Mathematical Society: $\$ 4.00$ per volume; single issues $\$ 1.50$. Back numbers are available.

Subscriptions, orders for back numbers, and changes of address should be sent to Pacific Journal of Mathematics, 103 Highland Boulevard, Berkeley, California, 94708.

PUBLISHED BY PACIFIC JOURNAL OF MATHEMATICS, A NON-PROFIT CORPORATION

Printed at Kokusai Bunken Insatsusha (International Academic Printing Co., Ltd.), 270, 3-chome Totsuka-cho, Shinjuku-ku, Tokyo 160, Japan. 


\section{Pacific Journal of Mathematics}

\section{Vol. 41, No. 2 December, 1972}

Tom M. (Mike) Apostol, Arithmetical properties of generalized Ramanujan sums .......................................... 281

David Lee Armacost and William Louis Armacost, On p-thetic groups ........ 295

Janet E. Mills, Regular semigroups which are extensions of groups .......... 303

Gregory Frank Bachelis, Homomorphisms of Banach algebras with minimal ideals ................................................ 307

John Allen Beachy, A generalization of injectivity .................. 313

David Geoffrey Cantor, On arithmetic properties of the Taylor series of rational functions. II.........................................

Václáv Chvátal and Frank Harary, Generalized Ramsey theory for graphs. III.

Small off-diagonal numbers .................................. 335

Frank Rimi DeMeyer, Irreducible characters and solvability of finite groups . . . . 347

Robert P. Dickinson, On right zero unions of commutative semigroups........ 355

John Dustin Donald, Non-openness and non-equidimensionality in algebraic

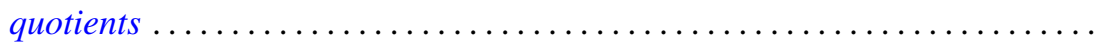

John D. Donaldson and Qazi Ibadur Rahman, Inequalities for polynomials with a prescribed zero ........................................ 375

Robert E. Hall, The translational hull of an $N$-semigroup ................ 379

John P. Holmes, Differentiable power-associative groupoids.............. 391

Steven Kenyon Ingram, Continuous dependence on parameters and boundary data for nonlinear two-point boundary value problems .

Robert Clarke James, Super-reflexive spaces with bases ..........

Gary Douglas Jones, The embedding of homeomorphisms of the plane in

continuous flows...............................

Mary Joel Jordan, Period $H$-semigroups and $t$-semisimple periodic

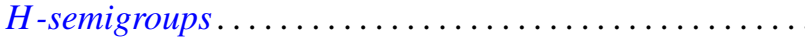

Ronald Allen Knight, Dynamical systems of characteristic 0

Kwangil Koh, On a representation of a strongly harmonic ring by sheaves...

Hui-Hsiung Kuo, Stochastic integrals in abstract Wiener space. ..

Thomas Graham McLaughlin, Supersimple sets and the problem of extending a

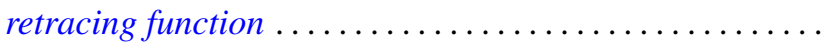

William Nathan, Open mappings on 2-manifolds .

M. J. O'Malley, Isomorphic power series rings

Sean B. O'Reilly, Completely adequate neighborhood systems and metrization

Qazi Ibadur Rahman, On the zeros of a polynomial and its derivative...

Russell Daniel Rupp, Jr., The Weierstrass excess function ..

Hugo Teufel, A note on second order differential inequalities and functional

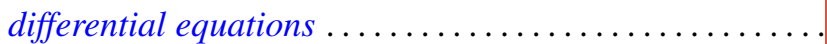

M. J. Wicks, A general solution of binary homogeneous equations over free 